anthropology \& materialism

\section{Anthropology \& Materialism}

A Journal of Social Research

$4 \mid 2019$

Art and Technique: A Framework of Unaccomplished Promises

\title{
Enfance berlinoise vers 1900 de Walter Benjamin
}

Une enfance remémorée selon la théorie de l'après-coup de Freud

Berlin Childhood Around 1900 by Walter Benjamin: The Recollection of a

Childhood According to Freud's Afterwardsness Theory

Infancia en Berlín hacia 1900 de Walter Benjamin: una infancia rememorada según la teoría del après-coup de Freud

\section{Olivier Taïeb}

\section{OpenEdition}

\section{Journals}

Édition électronique

URL : http://journals.openedition.org/am/948

DOI : $10.4000 / \mathrm{am} .948$

ISSN : 2364-0480

Éditeur :

CETCOPRA, CRASSH - Center for Research in the Arts Social Sciences and Humanities, Fakultät

Gestaltung - Universität der Künste Berlin

Référence électronique

Olivier Taïeb, "Enfance berlinoise vers 1900 de Walter Benjamin », Anthropology \& Materialism [En ligne], 4 | 2019, mis en ligne le 21 novembre 2019, consulté le 23 novembre 2019. URL : http:// journals.openedition.org/am/948 ; DOI : 10.4000/am.948

Ce document a été généré automatiquement le 23 novembre 2019.

Tous droits réservés 


\title{
Enfance berlinoise vers 1900 de Walter Benjamin
}

\author{
Une enfance remémorée selon la théorie de l'après-coup de Freud \\ Berlin Childhood Around 1900 by Walter Benjamin: The Recollection of a \\ Childhood According to Freud's Afterwardsness Theory \\ Infancia en Berlín hacia 1900 de Walter Benjamin: una infancia rememorada \\ según la teoría del après-coup de Freud
}

Olivier Taïeb

\section{Enfance berlinoise vers 1900 : une autobiographie fragmentée}

1 La genèse d'Enfance berlinoise vers 1900 de Walter Benjamin remonte à $1932^{1}$. Une revue littéraire propose à Benjamin d'écrire une série de textes sur Berlin. Mais ce contrat n'est pas honoré et le manuscrit, écrit principalement à Ibiza d'avril à août 1932, est abandonné, probablement en raison de la tournure personnelle que ce travail a fini par prendre. Il a été publié en 1970 par Gershom Scholem sous le titre Chronique berlinoise (Benjamin 2011b). A l'automne 1932, de retour à Berlin, Benjamin commence la rédaction d'Enfance berlinoise à partir de Chronique berlinoise mais en écartant les éléments qui se réfèrent trop directement à sa biographie réelle.

Enfance berlinoise n'est pas une œuvre qui a connu une édition définitive. Benjamin ne réussit pas à la publier de son vivant, alors qu'il y tient beaucoup et qu'il la considère comme très aboutie (Lavelle 2012, Lindner 2013). Il écrit à Scholem d'Italie le 25 octobre 1932 : «Je suis resté attelé à mon Enfance berlinoise vers 1900. J'ignore si quelque chose pourra en paraître bientôt, encore moins s'il existe quelque perspective de le publier sous forme de livre. Car pour autant que je puisse m'informer d'ici, mes travaux font actuellement en Allemagne l'objet d'un boycott " (Benjamin et Scholem 2010, 29). Certains textes sont d'ailleurs rédigés en français par Benjamin lui-même en 1933. Quelques chapitres sont parus sous forme de feuilleton dans la presse allemande entre 
1933 et 1935. En effet, en raison de la prise de pouvoir par les nazis, les éditeurs allemands refusent de s'engager dans des négociations pour la publication. Peu de temps avant son départ pour Paris en mars 1933, Benjamin vend le tapuscrit à la Frankfurter Zeitung qui en publie la plupart des pièces sous le pseudonyme de Detlev Holz.

Trois versions d'Enfance berlinoise ont été éditées. La première reprenant les parties publiées et deux cahiers manuscrits est l'édition établie en 1950 par Theodor W. Adorno, et traduite en français par Jean Lacoste en 1978 (Benjamin 2007). Elle comprend 41 textes et commence par le texte "Tiergarten ». Plus récemment, deux tapuscrits ont été découverts, composés par Benjamin lui-même et destinés à être publiés en livre. L'un a été découvert dans une bibliothèque universitaire à Giessen, en ex-RDA, en 1988. Il a été traduit en français par Pierre Rusch en 2012 (Benjamin 2012). Ce tapuscrit, rédigé en 1932 et en 1933, comprend 30 textes et commence par « La commerelle " (Mummerehlen). L'autre a été découvert par Giorgio Agamben à la Bibliothèque Nationale à Paris en 1981. Il aurait été finalisé en 1938 et en 1939. Il comprend aussi 30 textes mais commence par «Loggias ». Il a été traduit en français par Rusch en 2014 (Benjamin 2014). «Le Petit Bossu» est, lui, le texte conclusif de toutes les versions.

Enfance berlinoise est composée de textes brefs en prose, des "petits morceaux" proches des « images de pensée » d'un autre ouvrage de Benjamin, Sens Unique, publié en 1928 (Benjamin 2007) et d'autres textes publiés en 1933 (Benjamin 2011a). Dans une lettre à Scholem, le 26 septembre 1932 d'Italie, Benjamin évoque son manuscrit : « Il n'est pas seulement fort mince, il est aussi composé de petits morceaux : une forme à laquelle me ramène régulièrement d'abord la précarité matérielle de ma production, ensuite la considération de son exploitation commerciale. Dans le cas présent, il est vrai, cette forme me semble aussi absolument requise sur le plan objectif. Bref, il s'agit d'une série de notes, à laquelle je donnerai le titre Enfance berlinoise vers 1900 " (Benjamin et Scholem 2010, 27). Dans un texte sur Sens Unique, Adorno explique ce qu'est une image de pensée, terme utilisé à l'origine par le poète Stefan George à partir de Mallarmé :

[Les fragments de Sens Unique] ne veulent pas seulement en finir avec la pensée conceptuelle, ils veulent aussi choquer avec leur forme énigmatique et mettre ainsi en mouvement une pensée qui, dans sa forme conceptuelle traditionnelle, reste figée et donne alors l'impression d'être conventionnelle et désuète. Ce qu'on ne peut pas prouver dans le style ordinaire et dominant, cela doit stimuler la spontanéité et l'énergie de la pensée et mettre le feu aux poudres par une sorte de court-circuit intellectuel qui illumine les choses familières d'une nouvelle intensité (Adorno 2001b, 32).

5 Dans ces textes, la phénoménalité de la perception et la réflexion philosophique s'y trouvent étroitement liées (Lindner 2013). Pouvant être aussi comprise à partir du concept de Kant d'idée esthétique, l'image de pensée s'en éloigne parce qu'elle ne correspond pas seulement à l'illustration d'un contenu théorique (Lavelle 2014). La réflexion s'y immobilise de façon constructive et s'y fixe dans la limite topographique du texte lui-même. L'image de pensée n'est pas un récit, son objet n'est pas traité de façon narrative.

6 Dans Enfance berlinoise, la forme est proche mais le thème est ouvertement autobiographique. L'auteur reste cependant dissimulé même si Benjamin se résout à utiliser la première personne. Dans Chronique berlinoise, il raconte qu'il s'est fait une 
règle de ne jamais utiliser " je » dans ses écrits sauf dans ses lettres et que la qualité de son style est liée à cette règle. Le texte "Le manège", dont une version a été écrite pour Sens Unique, a gardé la troisième personne «l'enfant ». Dans la dernière version, celle de 1938-1939, Benjamin ajoute une préface qui en accentue le caractère autobiographique: «En 1932, alors que je me trouvais à l'étranger, je commençai à comprendre qu'il me faudrait bientôt dire adieu pour longtemps, pour toujours peutêtre, à la ville où je suis né " (Benjamin 2014, 19). Mais comme cet exil est dû à l'histoire, Benjamin espère que son récit aura des implications collectives, voire politiques :

C'est la raison pour laquelle les traits biographiques, qui se dessinent davantage dans la continuité que dans la profondeur de l'expérience, sont entièrement passés à l'arrière-plan dans ces tentatives. Et avec eux, les physionomies - celles de ma famille comme celles de mes camarades. [...] Les images de mon enfance citadine, en revanche, sont peut-être capables de préformer en leur sein une future expérience historique. J'espère en tout cas qu'on y verra combien celui dont il est question ici fut par la suite privé de la sécurité qui avait été accordée à son enfance (ibid., 20).

La succession des pièces n'est pas chronologique. Aucun texte ne peut être lu comme la suite de celui qui le précède. Toutefois, malgré cette discontinuité, les images se renvoient les unes aux autres selon leur titre, leur thème, leur lieu... En effet, ces images sont toutes spatialisées, ancrées à Berlin et dans ses alentours. Cette spatialisation est caractéristique de la «voie topographique» (Benjamin 2011b, 267) évoquée dans Chronique berlinoise :

Depuis longtemps, des années à vrai dire, je caresse l'idée d'organiser graphiquement sur une carte l'espace de la vie [...]. J'ai imaginé un système de signes conventionnels et sur le fond gris de telles cartes, on en verrait de toutes les couleurs si les logements de mes amis et amies, les salles de réunion des divers collectifs [...], les chambres d'hôtel et de bordel que j'ai connues le temps d'une nuit, les bancs décisifs du Tiergarten, les chemins de l'école et les tombes que j'ai vu remplir, les lieux où trônaient les cafés dont les noms ont aujourd'hui disparu et qu'on avait quotidiennement sur les lèvres, les courts de tennis où se trouvent aujourd'hui des maisons de rapport vides [...], si tout cela y était distinctement porté (ibid., 252).

7 Un réseau de correspondances entre les textes peut ainsi être identifié : «L'ensemble fonctionne comme une constellation de moments fixés, extraits de la progression temporelle, et spatialisés » (Lavelle 2014, 144). De cette façon, la remémoration de Benjamin se différencie de celle de Marcel Proust. Benjamin ne souhaite pas ouvrir «l'éventail du souvenir» :

Qui a commencé un jour à ouvrir l'éventail du souvenir trouve toujours de nouveaux segments, de nouvelles baguettes, aucune image ne lui suffit, car il a reconnu qu'on a beau le déplier, c'est dans les plis seulement que loge l'authentique, cette image, ce goût, cette impression tactile au nom de quoi nous avons déployé, déplié tout cela ; et alors le souvenir passe du petit au plus petit, du plus petit au minuscule [...]. Voilà le jeu mortel dans lequel se laissa entraîner Proust (Benjamin 2011b, 254).

8 Enfance berlinoise propose ainsi un autre infini, celui des fils associatifs et narratifs tissés par le lecteur entre ces fragments discontinus. 


\section{L'après-coup, l'espoir de remanier le passé}

9 Enfance berlinoise a été l'objet de nombreux commentaires. Parmi eux, l'article de Peter Szondi, «Espoir dans le passé », publié en 1961 et traduit en français une première fois en 1981 a constitué le début de la réflexion initiée ici. Szondi rappelle les relations de Benjamin avec l'œuvre de Proust. Benjamin n'en a pas été seulement un de ses premiers lecteurs en Allemagne, il en a été aussi le traducteur ${ }^{2}$ et le critique. Les relations intertextuelles entre Enfance berlinoise et A la recherche du temps perdu sont multiples (Kahn 2015). Mais Szondi souligne les divergences entre les deux auteurs à propos de la quête du temps perdu.

Dans A la recherche du temps perdu, ce qui est convoité par le narrateur n'est pas la simple remémoration du passé, c'est « quelque chose qui, commun à la fois au passé et au présent, est beaucoup plus essentiel qu'eux deux " (Proust 1986, 262). Avant un temps perdu retrouvé, c'est d'abord une expérience « extra-temporelle » qui est faite et qui permet "d'obtenir, d'isoler, d'immobiliser - la durée d'un éclair », ce qui n'est sinon jamais appréhendé: "un peu de temps à l'état pur»(ibid., 262). Dans ces moments-là, le narrateur devient un être « extra-temporel » indifférent à la mort : « Une minute affranchie de l'ordre du temps a recréé en nous pour la sentir l'homme affranchi de l'ordre du temps. Et celui-là on comprend qu'il soit confiant dans sa joie [...], on comprend que le mot de "mort" n'ait pas de sens pour lui ; situé hors du temps, que pourrait-il craindre de l'avenir?» (ibid., 263). Mais ces moments, qu'il n'est pas possible de faire advenir volontairement, sont très fragiles : «C'était peut-être bien des fragments d'existence soustraits au temps, mais cette contemplation, quoique d'éternité était fugitive " (ibid., 266). Comment alors les retrouver? Le retour sur les lieux du passé, expérimenté par le narrateur, n'est pas une solution. C'est l'écriture qui est "le seul moyen de retrouver le Temps perdu» (ibid., 293): "La vérité ne commencera qu'au moment où l'écrivain prendra deux objets différents, posera leur rapport, et les enchaînera par le lien indestructible d'une alliance de mots » (ibid., 283).

11 Il en est autrement pour Benjamin. Benjamin recherche dans le passé des moments, des lieux qui annoncent l'avenir, un avenir qui, depuis, est passé lui aussi. Il est ainsi attentif aux promesses faites à l'enfant qui ont été tenues ou non, aux prophéties et aux vœux qui se sont réalisés (comme dans "Matin d'hiver») ou non (comme dans «Loggias»). Benjamin peut ne pas raconter toute une vie et se consacrer juste à l'évocation de ces instants de l'enfance qui abritent une anticipation de l'avenir (Szondi 1981). Les lieux où sa mémoire entend retrouver quelque chose portent « les traces de ce qui allait venir» (Benjamin 2007, 60) (comme les endroits abandonnés du Parc Zoologique dans « La loutre »). Benjamin ne souhaite pas s'affranchir de la temporalité, il n'aspire pas à être anhistorique. Dans Enfance berlinoise, il rapporte plusieurs expériences de pertes irrévocables mais, en même temps, paradoxalement le passé n'est pas clos. Il s'agit d'une autre utopie que celle de Proust. Le passé peut se réinterpréter à la lumière du présent, c'est pour cela que le passé est ouvert, et peut ainsi changer. Benjamin recherche dans son enfance spécifiquement les moments du passé qui contiennent des traces qui seront secondairement comprises ou éclairées, voire modifiées ou réélaborées. C'est pour cela que le passé n'est pas dépourvu d'espoir selon lui.

12 Szondi cite un passage d'un texte de Sens Unique, "Madame Ariane, deuxième cour à gauche », qui résume magistralement le projet d'Enfance berlinoise : 
Lorsque à l'improviste le feu éclate ou que d'un ciel serein tombe la nouvelle d'une mort, dans le premier moment de terreur muette, un sentiment de culpabilité, de reproche informe : est-ce que tu ne le savais pas déjà ? Est-ce que la dernière fois que tu as parlé du mort, son nom avait déjà dans ta bouche une sonorité différente? Est-ce qu'au milieu des flammes tu ne perçois pas le signe d'hier soir, dont tu comprends maintenant seulement le langage? Et si un objet s'est perdu, que tu aimais, n'y avait-il pas déjà, des heures, des jours auparavant, un halo de dérision ou de deuil qui le trahissait? Le souvenir comme des rayons ultraviolets, révèle à chacun dans le livre de la vie une écriture qui, invisible, annotait comme une prophétie le texte (Benjamin 2007, 220).

Mais cette écriture invisible ne devient visible que dans un temps ultérieur. C'est tout à fait l'idée de l'après-coup de Freud. Benjamin est un grand lecteur de Freud. Le livre des passages, l'essai sur Baudelaire et Sur le concept d'histoire le montrent (Kleiner 1986). Mais même si probablement Benjamin connaissait la notion d'après-coup, il ne la cite pas explicitement, les principales analyses d'Enfance berlinoise non plus (Szondi 1981, Lacoste 2007, Lindner 1986, Witte 1986, Lavelle 2012, Kahn 2012, Lindner 2013).

L'après-coup apparait dans l'œuvre de Freud dès l'Esquisse d'une psychologie scientifique. Pour André Green (2000), l'après-coup est le temps inaugural de la théorie de la temporalité de Freud. Dans L'interprétation des rêves, apparaît déjà l'idée de la bidirectionnalité des processus psychiques, progrédiente et régrédiente. La dimension développementale de la temporalité se déploie ensuite avec les Trois essais sur la théorie sexuelle. Mais c'est avec l'Homme aux loups que l'après-coup occupe une place centrale (Green 2009). Selon cette idée, « des expériences, des impressions, des traces mnésiques sont remaniées ultérieurement en fonction d'expériences nouvelles, de l'accès à un autre degré de développement " (Laplanche et Pontalis 1994, 33). De nouveaux sens peuvent leur être donnés, mais l'après-coup n'est pas qu'une action différée. Il peut s'agir d'une véritable élaboration, d'un "travail de mémoire » (ibid., 36). L'après-coup peut être considéré comme « une dimension du psychisme mettant en jeu une mémoire activement transformatrice qui déborde ici de beaucoup la remémoration consciente " (Green 2000, 45). C'est, en effet, grâce à cette «mémoire transformatrice » que le passé peut se remanier et se transformer. C'est cette éventuelle transformation du passé, advenue, non advenue ou à advenir, qui est responsable de l'espoir dans le passé évoqué par Szondi à propos d'Enfance berlinoise, le passé si tragique soit-il n'étant jamais clos, mais toujours au moins entrouvert.

Ce texte, "Madame Ariane, deuxième cour à gauche", peut se rapprocher (comme un avant-coup ?) d'un passage du livre Le temps éclaté de Green :

Dans un premier temps - c'est le cas de le dire -, quelque chose se passe d'obscurément pressenti - mais à quoi cela ressemble-t-il ? - qui surgit et marque de son imprégnation la psyché. Cette matrice mal identifiable sera réveillée plus tard par un événement plus ou moins accidentel - mais non contingent - qui donnera cette fois une plus ample portée, un sens à valeur ajoutée, à ce qui fut reçu sous la forme d'une imprégnation investie d'une certaine potentialité significative, sans que puissent être précisés ni la force de celle-ci, ni le dessin qu'elle aura imprimé dans la psyché, ni sa visée conçue avec clarté, ni surtout quand et comment elle va refaire surface et à travers quels liens. Difficile de savoir au juste ce que la trace première « voulait dire » (ibid., 50).

Pour reprendre les termes de Benjamin, la prophétie de ce quelque chose « qui surgit et marque de son imprégnation la psyché » ne peut être «lue » que secondairement avec la survenue d'un événement ultérieur qui va lui donner sa direction précise. Parmi toutes les prophéties possibles, l'une sera choisie préférentiellement : 
L'analyse du terme allemand Nachträglich comporte deux idées. C'est d'une part, celle d'ultérieur et, d'autre part, celle de supplément. Autrement dit, entre deux événements psychiques I et II, le deuxième est reconnu dans son lien avec le précédent, auquel il donne maintenant un sens plus épanoui que sa trace mnésique première, isolée, ne laissait pas deviner. II donne donc à I, rétrospectivement, un sens qui n'existait qu'à l'état de virtualité mais que rien ne prédestinait à l'avance à prendre cette direction, parmi d'autres possibles dans le cadre d'une polysémie. La progression du sens comporte un retour en arrière qui accroît rétroactivement le contenu qu'il avait initialement et un choix «fixant» l'une parmi diverses possibilités (ibid., 50).

L'idée défendue ici est que c'est l'après-coup freudien qui donne au passé de l'espoir, l'espoir d'être mieux compris voire l'espoir d'être remanié ou réélaboré. Les résonances entre la notion d'après-coup et la remémoration dans Enfance berlinoise seront analysées à travers la lecture de quelques textes.

\section{Enfance berlinoise vers 1900 éclairée par la théorie de l'après-coup}

\section{Berlin : une ville labyrinthe}

Dans Enfance berlinoise, les remaniements après-coup sont souvent dus au développement de la sexualité, notamment post-pubertaire. Mais ils sont aussi secondaires au développement intellectuel, avec notamment la reconnaissance par la conscience de la mort comme fin de toute existence humaine.

Le plaisir que Benjamin a à se perdre dans une ville vue comme un labyrinthe est évoqué dans plusieurs textes, notamment «Tiergarten ». Le sens de ce plaisir n'est compris qu'ultérieurement avec le développement de la sexualité. Le premier temps est celui de l'école : «les premières traces furent des labyrinthes sur les buvards de mes cahiers » (Benjamin 2007, 29). Le terme " traces » rentre en résonance, bien sûr, avec la notion freudienne de trace mnésique. Ces traces dans le buvard, métaphore de la psyché, ont plusieurs potentialités mais le développement de la sexualité va leur donner une orientation principale. Tout labyrinthe a, en effet, son Ariane. Berlin l'a aussi pour Benjamin enfant : " Très tôt déjà j'en reçus un signe. C'est ici en effet, ou pas très loin, que doit avoir tenu son camp cette Ariane auprès de laquelle, pour la première fois et pour ne jamais plus l'oublier, je compris ce dont je ne connus que plus tard le nom : l'amour» (ibid., 30). Mais la mort va ravir l'objet de son amour, Louise de Landau ${ }^{3}$, et donnera un autre sens à cette quête labyrinthique : " Pourtant à sa source déjà apparaît la "Demoiselle" qui se posa plus tard, ombre glacée, sur lui [l'amour]» (ibid., 30). L'après-coup associe ici une troisième scène. Comme le dit Green, "rien n'oblige à limiter l'après-coup à deux scènes - comme la description première du cas Emma de l'Esquisse l'impliquait » (Green 2000, 50). Il peut mettre en chaîne différents sens « moins liés par une séquence que par une relation réticulée, le réseau d'ensemble permettant de situer les différents éléments qui se réverbèrent, se répondant au sein d'une structure arborescente indépendante des catégories qui règlent les ordres du temps et de l'espace » (ibid., 51). La mort de Louise de Landau est aussi évoquée dans «Deux énigmes»: «Elle s'appelait Louise de Landau et le nom m'avait bientôt ensorcelé. Aujourd'hui encore il reste vivant pour moi, mais ce n'est pas pour cette raison. Ce fut plutôt le premier nom parmi ceux du même âge sur lequel j'entendis 
tomber l'accent de la mort» (Benjamin 2007, 58). La connaissance de la mort par la conscience qui n'est pas acquise au début de l'enfance peut ainsi donner un autre sens à certains souvenirs comme dans « Le pupitre » :

J'aimais parcourir de vieux cahiers qui avaient une valeur tout à fait particulière car j'avais réussi à les protéger de la mainmise du maître qui avait la prétention de les conserver. Je laissais maintenant aller mon regard sur les corrections qu'il y avait portées à l'encre rouge et un plaisir tranquille me remplissait alors. Car, comme les noms des défunts sur la dalle funéraire qui désormais ne pourront jamais plus vous aider ou vous nuire, se trouvaient là les notes qui avaient dépensé toutes leurs forces sur des corrections antérieures (ibid., 99).

Un autre temps de l'enfance en rapport avec ce plaisir à se perdre est la lecture qui est aussi un labyrinthe. C'est un espace où il est agréable de se perdre et de " découvrir les corridors souterrains de ces histoires" (ibid., 94) comme dans un château (dans "Nouvel ami allemand de la jeunesse»). Dans deux autres textes, Berlin, ville labyrinthe, est encore plus explicitement associé à la sexualité. Dans "Eveil du sexe ", lors du Nouvel an juif, le narrateur est confié à un parent éloigné et il doit le rejoindre chez lui pour aller ensuite à la synagogue mais il se perd. Le sens de cette première transgression sera donné rétrospectivement par les expériences de l'adulte avec «la rue maquerelle » :

Alors que j'étais en plein désarroi, d'un seul coup, une vague brûlante d'angoisse m'envahit - "trop tard, c'est raté pour la synagogue" - et, avant qu'elle eût reflué, exactement au même instant, une autre survint, mais cette fois d'insouciance parfaite - "advienne que pourra, je m'en fiche". Et ces deux vagues unirent irrésistiblement leurs élans dans le premier grand sentiment de plaisir: la profanation du jour de fête s'associa à la rue maquerelle, qui me fit pressentir ici pour la première fois les services qu'elle devait rendre aux désirs adultes (ibid., 53).

21 Cette transgression marque aussi une séparation par rapport aux parents, déjà pressentie par l'enfant qui marche toujours derrière sa mère contre son avis (dans "Mendiants et prostituées »):

Je n'imaginais jadis la révolte que sous la forme du sabotage ; il est vrai que c'était par expérience personnelle. J'y recourais lorsque je tentais d'échapper à ma mère [...]. J'avais pris l'habitude en effet de rester toujours d'un demi-pas en arrière. C'était comme si je ne voulais en aucun cas aller de front, même avec ma propre mère. Tout ce que je devais à cette répugnance rêveuse lors de nos sorties communes en ville se révéla plus tard, lorsque le labyrinthe de celle-ci s'ouvrit au désir sexuel [...]. Autrefois déjà, cependant, lorsque ma mère blâmait encore mes répugnances et ma flânerie somnolente, j'entrevoyais obscurément la possibilité de me dérober un jour à sa tutelle grâce à la complicité de ces rues dans lesquelles je ne retrouvais apparemment pas mon chemin. Il n'est pas douteux en tout cas que le sentiment - malheureusement illusoire - d'échapper à ma mère, à sa classe et à la mienne, expliquait l'attrait sans exemple qui me poussait à aborder en pleine rue une prostituée (ibid., 108-9).

La relation avec sa mère est à nouveau explorée dans " Un fantôme ». L'enfant est en vacances avec ses parents à la campagne, près de Berlin. Il rêve d'un fantôme qui vole les « déshabillés » et les « soieries » de sa mère, rangés dans la réalité dans un endroit connu de lui mais inaccessible. Dans la nuit qui suit ce rêve, ses parents viennent se réfugier dans sa chambre parce que des cambrioleurs ont fait effraction dans la maison. Après le cambriolage, le lendemain, l'enfant raconte son rêve. Il s'en sent doublement coupable probablement parce que ce rêve trahit ses désirs œdipiens et aussi parce que s'il en avait parlé à temps, le cambriolage aurait pu, selon lui, être empêché : «Et en parlant je vis avec effroi que je n'aurais jamais dû raconter ce rêve » (ibid., 97). Le 
cambriolage donne au rêve un caractère prophétique et accentue après coup sa culpabilité vis-à-vis de ses fantasmes œdipiens. "Société » est, lui, un fragment qui évoque à nouveau sa mère et qui a une forte relation intertextuelle avec $A$ la recherche du temps perdu mais la séparation de l'enfant et de sa mère avant qu'elle ne parte à une soirée n'est pas ici source d'angoisse. L'enfant reçoit, le soir, quand sa mère vient lui souhaiter une bonne nuit, comme cadeau les heures que le jour a " encore en réserve " pour elle: «Je n'étais plus rempli à son départ que de l'orgueil de la laisser, aussi splendide, aller à la soirée. Et sans la connaître, je devinais dans mon lit, juste avant de m'endormir, la vérité d'une petite énigme : "Plus il est tard dans la soirée, plus beaux sont les invités." » (ibid., 76).

\section{Des énigmes à traduire et à élucider}

Plusieurs passages illustrent le modèle traductif de l'après-coup de Jean Laplanche (2006). «L'annonce d'une mort » en est le texte le plus éloquent. L'enfant a cinq ans, il est au lit, son père arrive pour lui dire bonne nuit. Il lui annonce la mort d'un cousin que l'enfant connaissait peu: «Je ne retins pas grand-chose de son récit. Mais je me suis bien imprégné de l'image de ma chambre et de mon lit, comme on prête davantage attention à un endroit dont on pressent qu'on devra un jour y aller chercher quelque chose d'oublié. C'est seulement après nombre d'années que j'appris quoi. Dans cette chambre mon père m'a tu une partie de la nouvelle. Le cousin était mort de syphilis » (Benjamin 2007, 54). L'adulte comprend plusieurs années plus tard pourquoi son père s'était tant attardé sur cette mort: «Mon père me donna la nouvelle avec tous les détails. Il décrit, à ma demande, ce qu'était une attaque cardiaque et il fut prolixe " (ibid., 54). Le message adressé par le père à l'enfant était énigmatique. L'enfant a pressenti qu'il s'agissait d'un message à traduire mais il n'y est parvenu que beaucoup plus tard. L'après-coup s'est joué ici dans une relation interpersonnelle, entre l'enfant et son père. Le message de l'adulte a constitué, comme le dit Laplanche, "l'avantcoup » de ce processus instituant chez l'enfant un déséquilibre qui le pousse à tenter de le traduire, en un second temps, dans l'après-coup. Le message est ainsi une énigme pour l'enfant :

Avec cette interrogation "Que me veut cet adulte? " s'ouvre tout le mouvement rétrogrédient de la traduction, en quête du secret du message énigmatique, mais qui pourtant le laissera toujours plus ou moins échapper. Tel est le mouvement à double direction de l'après-coup, chez tout être humain, donc pour chacun de nous. Le mouvement progrédient est celui du message énigmatique proposé originairement à l'enfant par l'adulte, comme étant «à traduire». Le mouvement rétrogrédient se situe dans les tentatives de traduction successives du récepteur, tout en sachant qu'on laissera toujours tomber quelque chose de l'énigmatique (Laplanche 2006, 171).

Le trouble suscité par cette énigme ressemble au trouble provoqué par la remémoration de relations amoureuses oubliées. Même si on a oublié une rencontre, on peut finir par «trébucher » sur son souvenir. La recherche par Benjamin du futur dans le passé consiste dans les tentatives de traduction, de résolution des énigmes, de quête de nouveaux sens. Il est nécessaire de retourner dans le passé, de façon rétrogrédiente pour élucider les messages laissés par l'adulte dans un mouvement progrédient :

On a souvent décrit le déjà vu. Est-ce que la formule est vraiment heureuse ? Ne faudrait-il pas parler d'événements qui nous parviennent comme un écho dont l'appel qui donna naissance semble avoir été lancé un jour dans l'obscurité de la vie 
écoulée. Au reste, à cela correspond le fait que le choc, par lequel un instant se donne à notre conscience comme déjà vécu, la plupart du temps nous frappe sous la forme d'un son. C'est un mot, un bruissement, un coup sourd qui a le pouvoir de nous appeler à l'improviste dans le tombeau glacial du passé, de la voûte duquel le présent semble nous revenir comme un simple écho. Etrange qu'on n'ait pas encore suivi le trajet inverse de cet éloignement - le choc par lequel un mot nous fait trébucher comme un manchon oublié dans notre chambre. De même que celui-ci conduit notre pensée vers une étrangère qui était là, il y a des mots et des silences qui conduisent notre pensée vers cette étrangère invisible : l'Avenir qui les oublia chez nous (Benjamin 2007, 53).

Mais l'énigme n'est pas toujours élucidée et peut entraîner la reprise du mouvement progrédient vers des synthèses approximatives et encore provisoires. Dans « Crimes et accidents ", le rêve raconté suscite toujours des interrogations :

Je ne sais plus si cette scène provient d'un rêve ou si seulement elle réapparut très souvent en rêve. [...] Dans le bureau de mon père il y avait un monsieur. Il n'est pas mal habillé. Il semble ne remarquer absolument pas la présence de ma mère. [...] La vie de mon père pend à un fil. [...] Mon père a la retraite coupée et l'autre n'a rien à faire avec ma mère. Ce qui est terrifiant chez lui, d'ailleurs, c'est cette manière de ne pas la voir, comme si elle était sa complice à lui, le meurtrier et le maîtrechanteur. Mais comme cette épreuve très sombre eut lieu sans me laisser le mot de son énigme, j'ai toujours compris celui qui cherche refuge auprès du premier avertisseur d'incendie venu (ibid., 117).

La complicité imaginée par l'enfant entre sa mère et le monsieur qui menace son père est l'élément le plus inquiétant. La trahison de sa mère vis-à-vis de son père est angoissante, surtout si l'inquiétude ressentie pour son père a une fonction de contreinvestissement d'une éventuelle agressivité contre lui.

L'énigme peut aussi être explicitement mise en scène par l'adulte comme M. Knoche, un des instituteurs de l'enfant, dans « Deux énigmes » :

M. Knoche demanda à la classe ce que pouvait bien signifier le dernier vers ${ }^{4}$. Naturellement personne ne put donner de réponse. Mais cela même sembla normal à M. Knoche, et il expliqua : "Vous comprendrez cela quand vous serez grands." [...] Plus tard [...] ce vers du "Chant des cavaliers" (maintenant que je le comprenais) ne recélait [pas] ce sens que $\mathrm{M}$. Knoche nous avait promis lors de la leçon de chant. La tombe vide et le cœur qu'on éprouve - deux énigmes dont la vie me devra encore la solution (ibid., 59).

L'enfant est devenu grand, mais le «portail aux vantaux fermés » ne s'est pas ouvert, contrairement à la promesse de M. Knoche : «Un portail aux vantaux fermés se dresse devant lui [l'enfant], et on lui assure qu'un jour celui-ci s'ouvrira pour lui sur le chemin de la vie. [...] Maintenant je suis grand. Je me trouve de l'autre côté du portail que M. Knoche nous montrait jadis. Mais ses vantaux sont toujours fermés. Je n'ai pas fait mon entrée par cette porte » (ibid., 67). Certaines promesses faites à l'enfant n'ont pas été tenues. L'adulte qu'il est devenu n'a pas eu de fonction institutionnelle reconnue, notamment universitaire, et a dû s'exiler à cause de l'avènement du IIIe Reich. D'autres promesses ont été tenues au-delà de toute mesure. Dans « Matin d'hiver », Benjamin se souvient, enfant, avoir formulé le souhait de ne pas avoir à se lever tôt le matin et ce souhait a été exaucé parce que Benjamin n'aura malheureusement pas de travail stable et régulier :

Chacun d'entre nous a cette fée qui accorde un vœu. Mais rares sont ceux qui savent se souvenir du souhait qu'ils formulèrent ; aussi, rares sont ceux qui reconnaissent plus tard dans leur propre vie leur vœu exaucé. Je sais celui qui pour moi se réalisa [...]. Une fois que j'étais arrivé là-bas [à l'école], il est vrai, au contact de mon banc, 
toute la fatigue qui me paraissait enfouie revenait décuplée. Et avec elle ce souhait: pouvoir dormir mon saoul. Je l'ai bien formulé mille fois et plus tard il fut exaucé réellement. Pourtant il fallut longtemps avant que je le reconnaisse exaucé, dans la vanité de tous mes espoirs d'avoir une situation et le pain assuré (ibid., 47).

\section{«Loggias » : le berceau et le mausolée de l'enfant}

Le texte intitulé «Loggias » est considéré par Benjamin comme son "autoportrait » (Benjamin et Scholem 2010, 90). Le 31 juillet 1933, il écrit à Scholem d'Ibiza : "Une nouvelle pièce, que j'ai ajoutée aux précédentes, m'a pendant un temps coupé de tout autre travail. Quelques pages sont nées sous le titre "Loggias", dont je ne puis rien annoncer que de très bon, en ajoutant qu'elles contiennent le portrait le plus exact qu'il m'ait été donné de faire de moi-même » (ibid., 81). Ce texte est placé au début par Benjamin dans sa version de 1938. La loggia de l'appartement de Berlin où Benjamin est né a été d'abord son «berceau » mais en est devenu son " mausolée », plus exactement le «mausolée » de l'enfant dans l'adulte, comme si en s'exilant, à l'âge adulte ("qui, pour ainsi dire, ne peut plus trouver nulle part de demeure » [Benjamin 2007, 122]) l'enfant en lui était mort définitivement. L'exil empêcherait en quelque sorte l'adulte de rester en relation avec l'enfant qu'il a été. L'enfant n'est pas parti, lui, et, ne pouvant plus survivre à l'intérieur de l'adulte, il doit alors inéluctablement mourir dans la ville de sa naissance: "C'est là que commence Berlin - le dieu de la ville lui-même. Il demeure là si présent que rien d'éphémère ne peut s'affirmer à ses côtés. Sous sa protection le temps et le lieu retrouvent leur identité et se rejoignent. Tous les deux se couchent ici à ses pieds. Mais l'enfant qui avait été jadis aussi dans le secret, maintenant enchâssé dans ce groupe, habite sa loggia comme un mausolée qui lui serait destiné depuis longtemps » (ibid., 122). Cette loggia est l'espace préparé dans l'Ouest de Berlin par ses parents, par ses grands-parents avant même sa naissance, c'est son « berceau ", mais un berceau où le temps va toujours plus vite que le développement de l'enfant, un temps qui va être ainsi générateur d'après-coup. « Une [loggia], ombragée l'été par des stores, fut le berceau dans lequel la ville déposa son nouveau citoyen. Les cariatides qui portaient la loggia de l'étage supérieur purent abandonner un instant leur faction pour chanter près de ce berceau une ballade qui, à vrai dire, ne contenait presque rien de ce qui m'attendait plus tard » (ibid., 119). L'avenir n'a pas tenu les promesses faites par les fées-cariatides. Mais cet espace est un véritable contenant de pensée pour Benjamin, pour ses rêves, ses écrits à venir, son intérêt pour « les images et les allégories ", pour ses amours : «L'air des cours m'a toujours depuis lors enivré. Je crois qu'une bouffée de cet air baignait encore ces vignes de Capri où je tenais embrassée ma bien-aimée; et c'est dans cet air-là que baignent les images et les allégories qui règnent sur ma pensée comme les cariatides des loggias sur les cours du vieil Ouest de Berlin » (ibid., 119). Mais cet espace protège de moins en moins l'enfant et laisse rentrer le monde extérieur avec le travail des domestiques, les tapis à battre, le linge à étendre ou le bruit du chemin de fer... La loggia montre des signes à l'enfant mais l'enfant ne sait pas encore les lire, il n'a pas encore les moyens de les rendre intelligibles. Ce n'est qu'après-coup que l'adulte se rendra compte que la mort était d'emblée omniprésente dans cette loggia :

Ici le printemps hissait les premiers bourgeons devant la façade grise de l'immeuble $\mathrm{du}$ fond ; et lorsque, plus tard dans l'année, une frondaison poussiéreuse mille fois par jour effleurait le mur de la maison, le frôlement des branches m'initiait à un savoir pour lequel je n'étais pas encore mûr. Car tout dans la cour me faisait signe. 
Combien de messages dans la fusillade des stores verts qu'on remontait! Et combien de dépêches funestes laissais-je prudemment fermées dans le grondement des rideaux de fer qui descendaient avec fracas dans le crépuscule (ibid., 119).

En interprétant rétrospectivement son expérience dans l'après-coup, Benjamin fait plus qu'un simple travail de remémoration consciente et ses souvenirs en sortent ainsi transformés.

L'impression d'être précédé, d'avoir été attendu est présente dans ce texte avec le berceau préparé mais cette impression peut persister et devenir inquiétante (dans le sens de l'inquiétante étrangeté de Freud) comme dans d'autres textes, dont «Le Petit Bossu » (voir plus loin) et « La lune » où l'enfant a peur que tout ait déjà eu lieu :

C'est alors que je levais la carafe avec la main pour verser l'eau dans un verre - tout cela frappait mon oreille comme une répétition. Car l'autrefois semblait avoir déjà occupé tous les endroits de cette terre parallèle où j'étais transporté. Aussi chaque son et chaque instant surgissaient-ils devant moi comme des doubles d'eux-mêmes. Et lorsque j'avais laissé ce sentiment s'emparer de moi pendant un instant, je m'approchais de mon lit tout empli de la crainte de m'y trouver moi-même déjà allongé (ibid., 130).

\section{Dans « Loggias » aussi, le temps s'accélère :}

Le temps vieillissait dans ces réduits ombreux qui s'ouvraient sur les cours. Et, pour cette raison même, lorsque je rencontrais le matin dans ces loggias, celui-ci était matin depuis si longtemps déjà qu'il semblait davantage lui-même que partout ailleurs. Et ainsi des autres moments de la journée. Jamais je ne pouvais les attendre ici. Ils étaient toujours là à m'attendre. Ils étaient là depuis longtemps déjà, et même pour ainsi dire déjà démodés, quand finalement je les y dénichais (ibid., 121). aussi celui de l'adulte est insuffisant pour comprendre les signes des catastrophes et des tragédies à venir. Le lecteur de la version d'Enfance berlinoise parue en 1950 et des suivantes en sait d'ailleurs plus que Benjamin, il sait qu'au bout de l'exil, Benjamin ne parviendra pas à quitter la France et se suicidera en 1940 à la frontière francoespagnole. Il sait aussi que la Shoah aura lieu. Par cette connaissance de l'histoire, la réception d'Enfance berlinoise est bien évidemment bouleversée dans un après-coup qui révèle les signes des morts à venir, certains de ces signes étant invisibles pour Benjamin lui-même. Adorno dans sa postface à Enfance berlinoise évoque à sa façon cet après-coup : «Les images qui nous rendent Berlin étonnamment proche ne sont $\mathrm{ni}$ idylliques ni contemplatives. L'ombre du Reich hitlérien plane déjà sur elles. Comme en rêve, ces images en épousent l'horreur bien avant qu'il soit né » (Adorno 2001a, 89).

Les histoires de vie sont ainsi ouvertes "par leurs deux extrémités» (Ricœur 1996, 191) : " La mémoire se perd dans les brumes de la petite enfance, ma naissance et, à plus forte raison l'acte par lequel j'ai été conçu appartient plus à l'histoire des autres, en l'occurrence celle de mes parents qu'à moi-même. Quant à ma mort, elle ne sera racontée que dans le récit de ceux qui me survivront ; je suis toujours vers ma mort, ce qui exclut que je la saisisse comme fin narrative » (ibid., 190). Comme Benjamin le dit lui-même dans ses Thèses sur l'histoire, "nous avons été attendus sur terre" (Benjamin 2003, 433) et précédés par nos parents et par les générations précédentes. Cette attente, énigmatique par nature, est « l'avant-coup » dont parle Laplanche. Green le rappelle dans ces termes: "Nous sentons que notre vie commence bien avant le moment où notre mémoire la fait débuter. S'il y a une inconscience de la mort pour l'inconscient, il y a aussi l'intuition que la vie ne coïncide pas avec la conscience et la mémoire que nous avons gardées du début de notre existence. Il serait presque plus 
juste de dire que, pour l'inconscient, l'existence n'a pas plus de fin qu'il ne peut lui assigner un début » (Green 2000, 170).

\section{« Le Petit Bossu » : la bosse de la mémoire et de l'oubli} pensé par Benjamin comme la pièce conclusive, équivalent de table des matières du livre. Benjamin reprend la chanson «Le Petit Bossu» lue dans le Deutsches Kinderbuch de Georg Scherer dont sa mère lui parlait quand il était enfant :

[Ma mère] parlait du Petit Bossu qui m'avait regardé. Celui que le Petit Bossu regarde ne fait pas attention. Ni à lui-même, ni même au Petit Bossu. Il se tient, effondré, devant un monceau de débris. [...] Là où il apparaissait je n'avais plus qu'à contempler les dégâts. Contemplation tardive, à laquelle les choses se dérobaient, jusqu'à ce que, en un an, le jardin fût transformé en jardinet, ma chambre en chambrette et mon banc en petit banc. Ils rapetissaient et c'était comme s'il leur poussait une bosse qui maintenant les intégrait eux aussi à l'univers du Petit Bossu, et pour très longtemps. Le bonhomme me devançait partout. Me devançant il me barrait le chemin. Mais autrement il ne faisait rien, ce prévôt, vêtu de gris, sinon toucher la moitié de l'oubli de chaque chose à laquelle je parvenais.

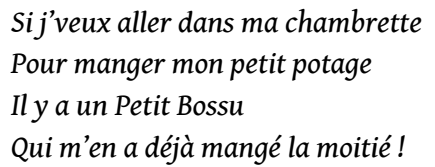

Le Petit Bossu était donc souvent là. Seulement je ne l'ai jamais vu. C'était toujours lui seul qui me regardait. Et plus son regard était perçant, et moins je me voyais moi-même (Benjamin 2007, 134).

Le Petit Bossu est un double de l'enfant. Il le précède, l'attend et l'observe tout le temps. Mais de cette observation continue, le Petit Bossu ne gardera en mémoire que quelques images, que quelques souvenirs, le reste sera oublié, le stigmate de cet oubli étant sa bosse. Dans son essai sur Franz Kafka, Benjamin associe le Petit Bossu à certains de ses personnages qui sont, eux aussi, « déformés » : «Ces personnages de Kafka sont liés par une longue série de figures à l'archétype de la déformation : au bossu. L'attitude que l'on rencontre le plus souvent dans les récits de Kafka est celle de l'homme qui incline profondément la tête sur la poitrine. [...] On voit clairement ici comme le fardeau va de pair avec l'oubli - l'oubli du dormeur. La même idée est exprimée dans la chanson populaire du Petit Bossu. » (Benjamin 2000a, 445). Dans le texte "La boîte à ouvrage », pour revenir à Enfance berlinoise, Benjamin fait référence à Odradek, autre figure ambiguë de la mémoire et de l'oubli. Odradek est une bobine de fil très singulière qui est, elle aussi, " déformée ». C'est un personnage du texte Le Souci du père de famille de Kafka : «Et je ne me serais guère étonné s'il y avait eu parmi les bobines une qui parlât, cette bobine Odradek, que je connus presque trente plus tard. Le poète [Kafka] appelle certes « le souci du père de famille » cette bobine mystérieuse qui parle et qui traîne dans les escaliers et dans les angles des pièces » (Benjamin 2007, 113).

Cette bosse est aussi un réservoir d'images inconscientes, peut-on dire en soulignant l'analogie avec la première topique freudienne. De là des souvenirs d'enfance peuvent revenir à la conscience de l'adulte. Le Petit Bossu n'est donc pas qu'un double de l'enfant. Il représente aussi la place occupée par l'adulte qui tente de se remémorer et qui « regarde » l'enfant qu'il a été. C'est, pourrait-on dire, «l'adulte dans l'enfant ». Lindner (2013) évoque à ce propos de façon très judicieuse le texte de Freud sur les 
souvenirs-écrans : « Dans la plupart des scènes d'enfance de quelque importance et par ailleurs non sujettes à caution, on voit, en se remémorant, sa propre personne apparaître en tant qu'enfant dont on sait qu'on est soi-même cet enfant; mais on voit cet enfant tel que le verrait un observateur se trouvant à l'extérieur de la scène " (Freud 2010a, 112). L'observateur, l'adulte, est à la place du Petit Bossu. Pour Freud, cet écart, entre l'enfant et l'adulte qui se souvient, est un signe de la réélaboration de la trace mnésique d'origine :

Quel que soit l'endroit où, dans un souvenir, sa propre personne se présente ainsi, objet parmi d'autres objets, on peut légitimement utiliser cette confrontation entre le moi qui agit et le moi qui se souvient comme preuve que l'impression d'origine a subi une réélaboration. C'est comme si une trace mnésique datant de l'enfance avait ici fait l'objet, à une époque postérieure (période de réveil), d'une traduction rétroactive en éléments plastiques et visuels. Mais rien de ce qui aurait été une reproduction de l'impression d'origine n'est jamais parvenu à notre conscience (ibid., 112).

Freud à la fin de son texte rapproche les souvenirs-écrans du reste des souvenirs d'enfance :

Peut-être est-il douteux, d'une façon générale, que nous ayons des souvenirs conscients provenant de notre enfance, et non pas, bien plutôt, qu'il s'agisse seulement de souvenirs relatifs à notre enfance. Nos souvenirs d'enfance nous montrent les premières années de notre vie, non pas telles qu'elles étaient, mais telles qu'elles nous sont apparues à des périodes de réveil ultérieures. Lors de ces périodes de réveil, les souvenirs d'enfance n'ont pas, comme on a l'habitude de le dire, émergé, mais ils ont alors été formés, et toute une série de mobiles qui visaient à atteindre bien autre chose que la vérité historique a exercé sa propre influence sur cette formation tout comme sur la sélection des souvenirs (ibid., 114).

L'adulte qui se remémore ne peut donc pas raconter ses souvenirs d'enfance du point de vue de lui enfant mais seulement du point de vue du Petit Bossu qui a observé l'enfant et qui en a recueilli les images les plus précieuses. Il s'agit donc pour l'adulte de récupérer ces images par le processus d'écriture, par l'associativité qui lui est nécessaire mais aussi par un effort de réélaboration et de construction (Freud 2010b). L'inconscient, qui a trouvé refuge dans cette précieuse bosse, est « un trésor de sens, réserve qui n'est pas seulement un conservatoire des illusions passées, mais une source dynamique inépuisable tendue vers le futur par la stimulation constante des forces qui délogent le sujet de son centre, le déplacent, le renvoient "ailleurs" » (Green 2000, 171). Le Petit Bossu est ainsi un témoin de la vie de l'enfant dont les images sont à retranscrire, à mettre en mots quand l'adulte tente de produire un texte autobiographique ou plus modestement quand il raconte sa vie. Le Petit Bossu est donc un double de l'auteur, c'est lui qui a organisé les images que le lecteur tient entre ses mains :

J'imagine que cette "vie tout entière » dont on raconte qu'elle passe devant les yeux des mourants, est composée d'images comme celles que le Petit Bossu a de nous tous. Elles défilent à toute allure comme ces pages des petits livres à la reliure serrée qui étaient jadis les précurseurs de nos cinématographes. Le pouce, en appuyant légèrement, avançait sur la tranche de ces petits livres [...]. Le Petit Bossu possède aussi des images de moi. Il me voyait dans ma cachette, et devant la cage de la loutre, le matin en hiver, et devant le téléphone dans le vestibule à l'arrière de la maison, et sur le mont de la Brasserie avec les papillons, et sur la patinoire au son de la fanfare, devant la boîte à ouvrage et penché sur mon tiroir, au Blumeshof et lorsque j'étais au lit, malade, à Glienicke et à la gare. Maintenant il en a terminé 
avec son travail. Pourtant sa voix [...] me chuchote encore ces mots par-dessus le seuil du siècle :

Ô mon enfant chéri, je t'en prie,

Prie aussi pour le Petit Bossu (Benjamin 2007, 135).

Enfance berlinoise se termine ainsi, sur une prière. Ce Petit Bossu, parfois malveillant, responsable des maladresses de l'enfant, oublieux le plus souvent, étrangement inquiétant donne cependant à l'adulte la possibilité de se remémorer quelques images de son enfance qu'il a lui-même sauvées de l'oubli. C'est pour cela qu'il faut prier pour lui.

\section{D'Enfance berlinoise à Sur le concept d'histoire : les implications politiques de la remémoration}

41 Pour finir, Enfance berlinoise est à lire aussi, comme Lacoste l'a proposé, à partir des conceptions sur l'histoire de Benjamin rédigées au début de l'année 1940 (Benjamin 2000 b et 2003).

Benjamin propose d'écrire l'histoire à l'envers, à partir du présent de l'historien qui " est tenu de brosser à contresens le poil trop luisant de l'histoire" (Thèse VII) (Benjamin 2003, 438). L'intelligibilité historique vient, en effet, « de la rencontre entre un moment du passé et un moment du présent, celui-là même où se situe l'historien » (Mosès 2006, 223). De cette rencontre entre ces événements non contigus "naît une figure de pensée nouvelle, où le présent féconde le passé et réveille le sens oublié ou refoulé qu'il porte en lui, alors que le passé retrouve, au cœur du présent, une actualité nouvelle " (ibid., 224). Benjamin transpose ainsi la bidirectionnalité des processus psychiques avec leurs mouvements rétrogrédients et progrédients au temps de l'histoire conçu comme une expérience intérieure collective. Il propose une archéologie matérielle et psychique à la recherche des traces laissées par le passé dans le présent (Didi-Huberman 2000). Il critique le temps continu accumulatif de l'idéologie du Progrès, construite par les Lumières et reprise ensuite par la pensée libérale et la pensée marxiste, pour défendre l'idée, empruntée au messianisme juif, d'une utopie surgissant au cœur même du présent, une utopie donnant une nouvelle chance à tout ce qui dans le passé a été manqué, oublié ou abandonné. Dans l'idéologie du Progrès défendue par les historiens des vainqueurs, l'avenir est prévisible, le Même revient inlassablement et les injustices se perpétuent (Löwy 2014). Face à ce danger, l'historien a une responsabilité importante. Il doit s'efforcer de sauver de l'oubli l'histoire des "vaincus $»^{5}$, "des sans-noms» (Benjamin 2003, 454) en ouvrant le passé, en le revisitant pour faire surgir quelque chose de nouveau :

C'est cette rupture de la temporalité historique, ce surgissement de l'imprévisible ${ }^{6}$ que Benjamin appelle du nom de Rédemption. Mais celle-ci n'est pas située quelque part à la fin des temps; au contraire, elle advient (ou elle peut advenir) à chaque instant, dans l'exacte mesure où chaque instant du temps - saisi dans sa singularité absolue - fait apparaître un nouvel état du monde. La différence qualitative de chacun des fragments du temps amène, avec elle, chaque fois du nouveau, la possibilité d'un changement imprévu (Mosès 2006, 215).

Tout ce qui dans le passé, tant personnel que collectif, a été manqué, a échoué ou n'a pas abouti, peut être ainsi réélaboré et remanié dans le présent, de même que tout ce qui a été oublié peut être rappelé à la mémoire. 
C'est, en effet, à partir des espoirs des générations passées que les rêves prennent forme pour Benjamin. L'utopie dépend ainsi de la mémoire :

C'est donc à nous de nous rendre compte que le passé réclame une rédemption dont peut-être une toute infime partie se trouve être placée en notre pouvoir. Il y a un rendez-vous mystérieux entre les générations défuntes et celle dont nous faisons partie nous-mêmes. Nous avons été attendus sur terre. Car il nous est dévolu à nous comme à chaque équipe humaine qui nous précéda, une parcelle du pouvoir messianique. Le passé la réclame, a droit sur elle. Pas moyen d'éluder sa sommation. L'historien matérialiste en sait quelque chose (Thèse II) (Benjamin 2003, 434).

Il est notamment « intimement persuadé que, si l'ennemi triomphe, même les morts ne seront pas en sûreté $»^{7}$ (Thèse VI) (Benjamin 2000b, 431). Pour l'historien, la remémoration ne se contente donc pas seulement d'évoquer un moment du passé, elle vise à le transformer. Grâce à elle, le temps historique cesse d'apparaître comme irréversible. Il est possible d'intervenir pour en changer dans l'après-coup sa signification. L'après-coup met en jeu, comme le dit Green, « une mémoire activement transformatrice». C'est, en quelque sorte, le travail de mise en mémoire du Je, apprenti-historien décrit par Piera Aulagnier (2000). Dans ce processus, il s'agit à nouveau de déchiffrer les traces que le passé a laissées dans le présent : «Le passé a laissé de lui-même des images comparables à celles que la lumière imprime sur une plaque photosensible. Seul l'avenir possède des révélateurs assez actifs pour fouiller parfaitement de tels clichés » (Benjamin 2003, 452). Ce qui est très proche du texte, «Madame Ariane, deuxième cour à gauche ", cité plus haut.

Benjamin propose ainsi une histoire où chaque moment du passé peut être réactualisé, rejoué dans d'autres conditions, sur une nouvelle scène. Le messianisme n'est pas conçu comme l'attente d'une apothéose qui se produirait au terme d'un temps linéaire et continu mais comme la possibilité donnée à chaque moment du temps, de l'avènement du nouveau.

Pour revenir à Enfance berlinoise, la solidarité qui existe entre les générations existe aussi entre l'enfant et l'adulte chez un sujet donné (Lacoste 2007). L'historien doit redonner la parole aux oubliés, aux vaincus de l'Histoire et l'adulte doit avoir la même préoccupation vis-à-vis de l'enfant qu'il a été et vis-à-vis de l'enfant qui est toujours en lui : «Enfance berlinoise doit peut être son existence à cette étrange et belle idée théologico-politique: nous avons envers l'enfant mort qui est en nous la même responsabilité qu'envers les espérances toujours en souffrance du passé » (ibid., 13).

Comme les historiens, les psychanalystes tentent ainsi d'exercer leur "parcelle de pouvoir messianique » pour que leurs patients restent en relation avec l'enfant qu'ils ont été tout en essayant de passer d'une répétition mortifère à une remémoration génératrice de changements dans le présent mais aussi dans le passé. Tel est peut-être un des enseignements d'Enfance berlinoise et des Thèses : « sauver de l'oubli » l'ambition révolutionnaire de la psychanalyse et révéler, dans l'après-coup, la dimension politique de la théorie de la temporalité de Freud. 


\section{BIBLIOGRAPHIE}

Adorno, Theodor W. 2001a [1950]. « Postface à une enfance berlinoise au XIXe siècle » In Sur Walter Benjamin. Trad. Christophe David, 88-91. Paris : Gallimard, coll. Folio essais.

Adorno, Theodor W. 2001b [1955]. « Sens Unique » In Sur Walter Benjamin. Trad. Christophe David, 31-9. Paris : Gallimard, coll. Folio essais.

Aulagnier, Piera. 2000 [1984]. L'apprenti-historien et le maître sorcier. Du discours identifiant au discours délirant. Paris : PUF.

Benjamin, Walter. 2000a [1934]. « Franz Kafka. Pour le dixième anniversaire de sa mort ». Trad. Maurice de Gandillac revue par Pierre Rusch. In Euvres II, 434-46. Paris : Gallimard, coll. Folio essais.

Benjamin, Walter. 2000b [textes rédigés en 1940 selon les éditeurs de ses œuvres]. « Sur le concept d'histoire » Trad. Maurice de Gandillac revue par Pierre Rusch. In Fuvres III, 427-43. Paris : Gallimard, coll. Folio essais.

Benjamin, Walter. 2003 [textes rédigés en 1940 selon les éditeurs de ses œuvres]. « Sur le concept d'histoire » In Ecrits français, 423-55. Paris : Gallimard, coll. Folio essais.

Benjamin, Walter. 2007 [textes rédigés entre 1927 et 1935 selon les éditeurs de ses œuvres]. Sens unique précédé de Une enfance berlinoise et suivi de Paysages urbains. Trad. Jean Lacoste. Paris : Les lettres nouvelles - Maurice Nadeau.

Benjamin, Walter. 2011a [textes rédigés entre 1925 et 1935 selon les éditeurs de ses œuvres]. Images de pensée. Trad. Jean-François Poirier et Jean Lacoste. Paris : Christian Bourgois.

Benjamin, Walter. 2011b [textes rédigés en 1932 selon les éditeurs de ses œuvres]. « Chronique berlinoise » In Ecrits autobiographiques. Trad. Christophe Jouanlanne et Jean-François Poirier, 249-336. Paris : Christian Bourgois.

Benjamin, Walter. 2012. [textes rédigés entre 1932 et 1933 selon les éditeurs de ses œuvres]. Enfance berlinoise vers 1900. Version dite de Giessen (1932 - 1933). Trad. Pierre Rusch. Paris : L'Herne.

Benjamin, Walter. 2014 [textes rédigés entre 1938 et 1939 selon les éditeurs de ses œuvres]. Enfance berlinoise vers 1900. Trad. Pierre Rusch. Paris : Hermann.

Benjamin, Walter et Scholem, Gershom. 2010 [1933-1940]. Théologie et utopie. Correspondance 1933-1940. Trad. Didier Renault et Pierre Rusch. Paris : L'éclat.

Brodersen, Momme. 2013. «Qui était Luise von Landau? A propos d'une figure d'Enfance berlinoise ». Trad. Didier Renault. In Cahier Walter Benjamin, édité par Patricia Lavelle, 106-11. Paris : L'Herne.

Didi-Huberman, Georges. 2000. Devant le temps. Paris : Minuit.

Freud, Sigmund. 1979 [1918]. « Extrait de l'histoire d'une névrose infantile. L'homme aux loups ». In Cinq psychanalyses. Trad. Marie Bonaparte et Rudolph M. Loewenstein, 325-420. Paris : PUF.

Freud, Sigmund. 2010a [1899]. « Sur les souvenirs-écrans ». In Huit études sur la mémoire et ses troubles. Trad. Denis Messier, 77-116. Paris : Gallimard.

Freud, Sigmund. 2010b [1937]. « Constructions en analyse ». In Huit études sur la mémoire et ses troubles. Trad. Denis Messier, 165-90. Paris : Gallimard. 
Green, André. 2000. Le temps éclaté. Paris : Minuit.

Green, André. 2009. « L'après-coup dans la théorie de la temporalité. Le cas de l'Homme aux loups ». Revue française de Psychanalyse 73 : 1495-502.

Kafka, Franz. 2012 [1919]. « Le Souci du père de famille ». In Un artiste de la faim, A la colonie pénitentiaire et autres récits. Trad. Claude David, 139-41. Paris : Gallimard, coll. Folio.

Kahn, Robert. 2012. «Walter Benjamin ». In Poétiques du récit d'enfance, édité par Isabelle Poulin, Robert Kahn, Evelyne Thoizet, 17-57. Neuilly : Atlande.

Kahn, Robert. 2015. « Présentation ». In Walter Benjamin, Sur Proust. Trad. Robert Kahn, 7-35. Caen : Nous.

Kleiner, Barbara. 1986. «L'éveil comme catégorie centrale de l'expérience historique dans le Passagen-Werk de Benjamin ». In Walter Benjamin et Paris, édité par Heinz Wismann, 497-515. Paris : Les Editions du cerf.

Lacoste, Jean. 2007 [1978]. « Préface ». In Walter Benjamin, Sens unique précédé de Une enfance berlinoise et suivi de Paysages urbains. Trad. Jean Lacoste, 9-24. Paris : Les lettres nouvelles Maurice Nadeau.

Laplanche, Jean. 2006 [1990-1991]. Problématiques VI. L'après-coup. Paris : PUF.

Laplanche, Jean et Pontalis, Jean-Bertrand. 1994 [1967]. « Après-coup ». In Vocabulaire de la psychanalyse, 33-6. Paris : PUF.

Lavelle, Patricia. 2012. «Préface ». In Walter Benjamin, Enfance berlinoise vers 1900. Version dite de Giessen (1932 - 1933). Trad. Pierre Rusch, 9-25. Paris : L'Herne.

Lavelle, Patricia. 2013. «Les forces anticipatrices de l'œuvre littéraire : sur l'espoir dans le passé ». Revue Germanique Internationale 17, 115-32.

Lavelle, Patricia. 2014. « Post-scriptum. Les mues d'Enfance berlinoise ». In Walter Benjamin, Enfance berlinoise vers 1900. Trad. Pierre Rusch, 143-51. Paris : Hermann.

Lindner, Burckhardt. 1986. « Le Passagen-Werk, Enfance berlinoise et l'archéologie du passé le plus récent ». In Walter Benjamin et Paris, édité par Heinz Wismann, 13-32. Paris : Les Editions du cerf.

Lindner, Burckhardt. 2013. «L'enfant remémoré. Sur la forme poétique et la structure mémorielle d'Enfance berlinoise vers 1900 ». In Cahier Walter Benjamin, édité par Patricia Lavelle, 97-105. Paris : L'Herne.

Löwy, Michael. 2014. Walter Benjamin : Avertissement d'incendie. Une lecture des Thèses «Sur le concept d'histoire ». Paris : L'éclat.

Mosès, Stéphane. 2006 [1992]. L’Ange de l'Histoire. Rosenzweig, Benjamin, Scholem. Paris : Gallimard, coll. Folio essais.

Proust, Marcel. 1986 [1927]. Le Temps retrouvé. Paris : Flammarion, coll. GF.

Ricœur, Paul. 1996 [1990]. Soi-même comme un autre. Paris : Seuil, coll. Points Essais.

Szondi, Peter. 1981 [1961]. « Espoir dans le passé. A propos de Walter Benjamin ». Trad. Sabine Bollack. In Poésies et poétiques de la modernité, 33-48. Lille : Presses Universitaires.

Traverso, Enzo. 1996. « Auschwitz "ante" : Benjamin et l'Angelus Novus ». Europe, n804, « Walter Benjamin » : 169-79. 
Witte, Bernd. 1986. « Paris - Berlin - Paris. Des corrélations entre l'expérience individuelle, littéraire et sociale dans les dernières œuvres de Benjamin ». In Walter Benjamin et Paris, édité par Heinz Wismann, 50-62. Paris : Les Editions du cerf.

\section{NOTES}

1. Walter Benjamin est né à Berlin en 1892. Il y a vécu jusqu'à son départ pour Paris en mars 1933 après l'accession d'Hitler au pouvoir. Il n'y retournera plus. Il tentera de passer en Espagne, mais face à l'échec de cette tentative, il se suicide le 26 septembre 1940 à Port-Bou.

2. Benjamin a traduit A l'ombre des jeunes filles en fleur et Du côté de Guermantes avec Franz Hessel.

3. Louise de Landau n'est pas décédée quand Benjamin écrit Enfance berlinoise, elle sera assassinée à Auschwitz en 1944 (Brodersen 2013).

4. Le vers (Là, c'est encore le cour qu'on éprouve!) fait partie du chant des cavaliers dans Le camp de Wallenstein de Schiller.

5. Benjamin se considère comme un «vaincu » de l'histoire, comme il le rappelle dans la Thèse XII : « Notre génération à nous est payée pour le savoir, puisque la seule image qu'elle va laisser est celle d'une génération vaincue. Ce sera là son legs à ceux qui viennent » (Benjamin 2003, 440). 6. La loutre du Parc Zoologique de Berlin dans Enfance berlinoise, dont l'apparition, imprévisible, est précédée d'une longue attente, illustre la discontinuité de l'expérience temporelle (Lavelle 2013).

7. A nouveau, l'œuvre de Benjamin des années 1930, lue après la Shoah, se charge dans l'aprèscoup d'une puissante valeur prémonitoire (Traverso 1996).

\section{RÉSUMÉS}

Enfance berlinoise vers 1900 est le projet autobiographique de Walter Benjamin. Sa rédaction s'est étendue entre 1932 et 1939. Il est composé de textes brefs qui tissent entre eux un réseau de correspondances. Dans ces textes, Benjamin s'intéresse à l'avenir dans le passé, c'est-à-dire aux moments du passé qui annoncent l'avenir, que cet avenir soit advenu ou non advenu depuis. Il est ainsi attentif à "l'espoir dans le passé» (selon l'expression de Peter Szondi). Il recherche électivement dans son enfance les traces qui ont été ultérieurement comprises, remaniées ou réélaborées. Cet article montre que cette remémoration est une illustration exemplaire de l'idée de l'après-coup de Freud. Benjamin, d'ailleurs, transposera à l'échelle de l'histoire ce travail de la mémoire dans ses Thèses, rédigées avant sa mort en 1940, et donnera ainsi, en retour, un sens politique à la théorie de la temporalité de Freud.

Berlin Childhood Around 1900 is Walter Benjamin's autobiographical project. It was written between 1932 and 1939. It consists of short pieces, intertwined by a network of correspondences. In these pieces, Benjamin looks at the future in the past, that is to say, at moments of the past that foreshadow the future, whether this future has occurred or not. Benjamin thus pays close attention to «hope in the past» (as Peter Szondi puts it). In his childhood, he carefully looks for traces that have later been understood, rearranged or further elaborated. This article shows that this recollection is an exemplary illustration of Freud's idea of afterwardsness. It is worth noticing that Benjamin later transposed this work of recollection to the realm of history, in his 
Theses written before his death in 1940, thus confering a political significance to Freud's theory of temporality.

Infancia en Berlín hacia 1900 es el proyecto autobiográfico de Walter Benjamin. Redactado entre 1932 y 1939, se compone de textos breves que tejen entre sí una red de correspondencias. Estos textos reflejan el interés de Benjamin por el futuro del pasado, es decir, por los momentos del pasado que anuncian el porvenir, aunque este no se haya finalmente realizado desde aquel entonces. En este sentido, Benjamin presta atención a "la esperanza en el pasado" (según la expresión de Peter Szondi), buscando electivamente en su infancia las trazas que fueron comprendidas, remodeladas o reelaboradas ulteriormente. El presente artículo muestra que esta rememoración es una ilustración ejemplar de la idea del après-coup de Freud. Por lo demás, Benjamin trasladará a la escala de la historia este trabajo de la memoria en sus Tesis, redactadas antes de su muerte en 1940, dando así, retrospectivamente, un sentido político a la teoría de la temporalidad de Freud.

\section{INDEX}

Keywords : Walter Benjamin, Berlin Childood Around 1900, afterwardsness, recollection Palabras claves : Walter Benjamin, Infancia en Berlín hacia 1900, après-coup, rememoración Mots-clés : Walter Benjamin, Enfance berlinoise vers 1900, après-coup, remémoration

\section{AUTEUR}

\section{OLIVIER TAÏEB}

Psychiatre, Praticien Hospitalier, Service de Psychopathologie de l'enfant et de l'adolescent, de Psychiatrie générale et d'Addictologie (Pr T. Baubet), Hôpital Avicenne, APHP, 129 rue de Stalingrad, 93000 Bobigny, Université Paris 13, CESP Inserm U1178 